\title{
Associations between adolescents' experience of general practitioners and health outcomes in England: a cross-sectional study of national data
}

\author{
Arrash A Yassaee, Dougal S Hargreaves, Stephanie Lamb, Kayleigh Chester, Fiona M Brooks
}

Published Online November 13, 2015 UCL Institute of Child Health, London, UK (A A Yassaee MBBS, D S Hargreaves MD); Herne Hill Group Practice and The Well Centre, London, UK (S Lamb MBBS); and Centre for Research in Primary and Community Care, University of Hertfordshire, Hatfield, UK (K Chester BSc, Prof F M Brooks PhD)

Correspondence to: Dr Arrash A Yassaee, UCL Institute of Child Health, 30 Guilford Street, London WC1N 1EH, UK arrash@cantab.net

\section{Abstract}

Background Adolescence is a key stage of the life course when lifelong health behaviours and attitudes to health care can be established. Poor experience of consultations with a general practitioner (GP) is common among adolescents, but little is known about whether poor experience in this group is associated with worse health status or outcomes. This study aimed to investigate this association with data from the 2014 Health Behaviour in School-Aged Children (HBSC) survey (England).

Methods We used logistic regression to analyse data for 5335 participants aged 10-17 years in the HBSC survey. Four aspects of recent GP experience were studied: feeling at ease, being treated with respect, quality of GP explanation, and feeling able to talk about personal matters. Five dichotomised measures of health status or outcome were used (ever self-harmed; fair or poor self-reported health; frequent [at least weekly] low mood, sleeping problems, or headaches). We adjusted for participants' sex, age, ethnicity, and family affluence score. Of 5178 participants, 1187 (23\%) had not visited their GP within the past year and were excluded from the analysis.

Findings Of 3991 adolescents, 3632 (91\%) felt treated with respect, 2091 (52\%) could talk about personal matters, $1600(40 \%)$ were satisfied with explanations, and 1221 (31\%) felt at ease. Participants who reported poor experience of GP care were more likely to have poor health outcomes than were those who reported a good experience. For example, adolescents who did not feel at ease with their GP were more likely to report self-harm (adjusted odds ratio [AOR] $2 \cdot 65$, 95\% CI 1.69-4.15; fair or poor health 1.64, 1.28-2.10; low mood 1.51, 1.25-1.82) and sleeping problems $(1.41$, 1.19-1.66). All GP indicators were associated with self-harm (AOR range 1.64-2.70; quality of GP explanation $\mathrm{p}=0.006$, all others $\mathrm{p}<0 \cdot 001)$ and feeling low $(1 \cdot 46-2 \cdot 11$, all $\mathrm{p}<0 \cdot 001)$. The association with GP experience was less consistent for the other three health outcomes.

Interpretation This cross-sectional, observational study demonstrates that young people who report worse health symptoms, typically also report a poor experience of care. Further research is needed to investigate whether GP experience influences health outcomes-suggesting that improving GP experience might improve health outcomes in this group—or whether poor health status leads to more negative perceptions of care.

Funding None.

Contributors

DSH, FMB, and AAY conceived the study. AAY performed all analyses and wrote the first draft of the abstract. All authors critically reviewed and revised the abstract.

Declaration of interests

We declare no competing interests.

Acknowledgments

HBSC is a WHO collaborating study. HBSC England is funded by the Department of Health for England. 